\title{
Datenschutz-Grundverordnung: Neuer Schutz - neue Spannungsverhältnisse
}
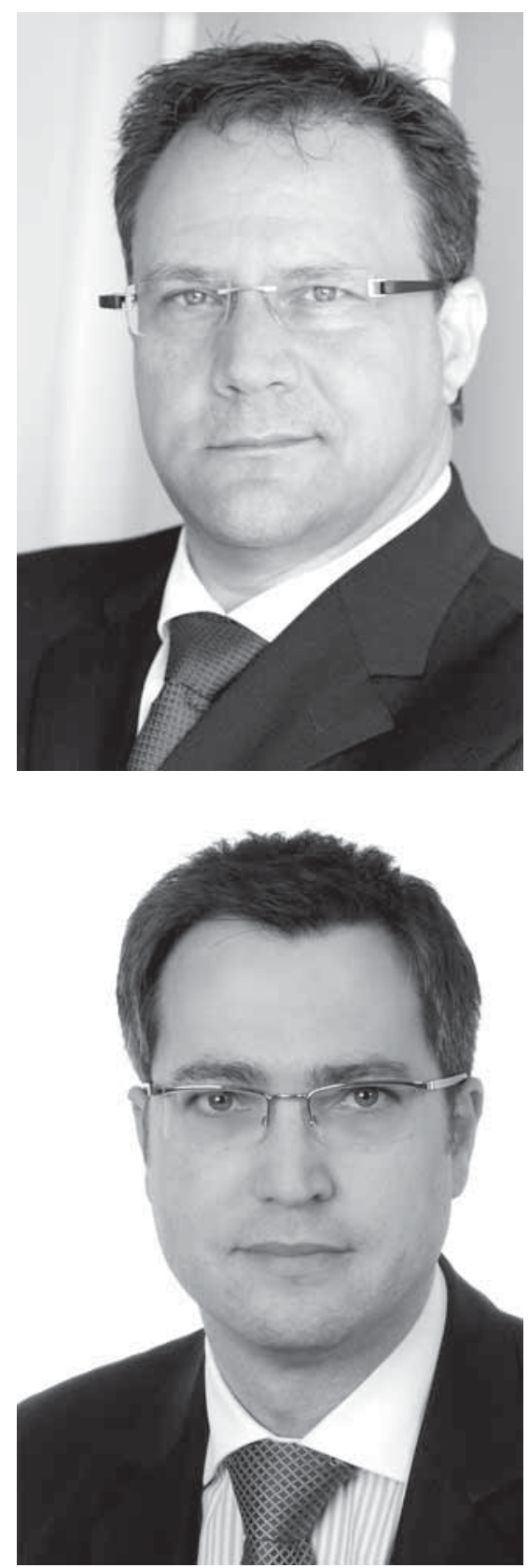

Die Datenschutz-Grundverordnung ist ein Meilenstein von bisher nicht da gewesener Größe im Datenschutzrecht der EU. Sie fußt auf Art. 8 der Charta der Grundrechte der Europäischen Union. Als Verordnung ist sie unmittelbar in allen EU-Mitgliedsstaaten anwendbar und setzt einheitliche Maßstäbe. Entscheidender ist aber, was häufig unterschätzt wird, dass sie mit ihren Regelungen alle Bereiche der Verarbeitung personenbezogener Daten in Unternehmen und öffentlichen Stellen durchdringt, weshalb mit nur sektoralen oder partikularen Anpassungen der Datenverarbeitung die Vorgaben der Datenschutz-Grundverordnung nicht umgesetzt werden können.

Gleichzeitig ist dies eine der Problemlagen bei der Umsetzung und Erfüllung der Vorgaben der Datenschutz-Grundverordnung in der Praxis. Denn ihren Regelungen ist eine erhebliche Abstraktion immanent, da sie eben nicht nur partikulare oder sektorale Aspekte regelt. Ihre Auslegung und Anwendung kann - was erschwerend hinzukommt - nicht aus einem nationalen Verständnis heraus in den jeweiligen Mitgliedsstaaten erfolgen, sondern muss unionsweit einheitlich ausgelegt und angewendet werden. Auch kommt hinzu, dass der Trilog im Rahmen des EU-Gesetzgebungsverfahren die strukturelle und systematische Einheit eines Gesetzes nicht unbedingt fördert.

Dem eingangs angesprochenen großen Ziel der Datenschutz-Grundverordnung muss also Zeit gegeben werden. Es wird sich wohl erst in einigen Jahren beurteilen lassen, ob die Datenschutz-Grundverordnung der "große Wurf" ist. Sicher ist aber, dass diesem neuen Datenschutzrecht seit Inkrafttreten der Datenschutz-Grundverordnung am 25. Mai 2016 und auch noch einige Jahre nach ihrem Anwendungsbeginn am 25. Mai 2018 Unklarheiten in Bezug auf Auslegung und Anwendung immanent sind. Dies führt zur Rechtsunsicherheit für den Rechtsanwender.

Verschärft wird dieses Gefühl der Unsicherheit durch die gewollt hohen Sanktionen der Datenschutz-Grundverordnung bei Verstößen. Böse Zungen könnten formulieren, dass dieser Verschärfung eine Lernkurve des Gesetzgebers - möglicherweise auch aus anderen Rechtsgebieten - zugrunde liegt: Ist die Sanktion hoch genug, ist auch die Beachtung der gesetzlichen Regelungen entsprechend hoch. Doch auch wenn dies scheinbar in jüngerer Zeit manchen zu überraschen bzw. zu verwundern scheint, datenschutzrechtliche Verbote, Anforderungen und Pflichten gibt es auf EU-Ebene bereits seit 1995 und auf nationaler Ebene sogar noch länger. Der Gesetzgeber dürfte im Falle der Datenschutz-Grundverordnung jedenfalls eines seiner Ziele - nämlich Aufmerksamkeit für den Datenschutz - schon jetzt erreicht haben.

Mit dem Ausbau der Sanktionen führt der Gesetzgeber jedoch neben dem vorgenannten stärkeren Schutz der betroffenen Personen, auch neue Spannungsverhältnisse in den Datenschutz ein, indem er den Sanktions- und Haftungsdruck in den Vordergrund rückt. Die Bewertung von datenschutzrechtlichen Fragestellungen wird damit stärker als bisher mit der Frage nach Haftung und Sanktion verbunden sein. Das wird eventuell auch die Kommunikation mit Aufsichtsbehörden belasten, wenngleich das wohl von keiner Seite gewollt ist. Darüber hinaus stellt sich für die Handelnden ebenso die Frage nach der persönlichen Haftung - sowohl für die Organe der Unternehmen (direkte und mittelbare Haftungen), als auch für die in Unternehmen für die Verarbeitung der personenbezogenen Daten zuständigen Mitarbeiter sowie den Datenschutzbeauftragten.

Es steht zu erwarten, dass jede datenschutzrechtliche Fragestellung zukünftig mit der Frage nach der Sanktion und der Haftung hierfür verbunden sein wird. Gerade die durch die Datenschutz-Grundverordnung vorgesehenen Melde- und Benach- 


\section{Ergänzt die Zeitschrift DuD - Datenschutz und Datensicherheit} Die Buchreihe für den interdisziplinärer Dialog

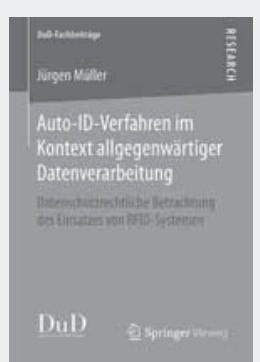

2018, XXXI, 652 S.

6 Abb., 4 Abb. in Farbe. Brosch.

$€(D) 79,99|€(A) 82,23|$

${ }^{*} \mathrm{sFr} 82,50$

ISBN 978-3-658-19124-5

Auto-ID-Verfahren im Kontext allgegenwärtiger Datenverarbeitung

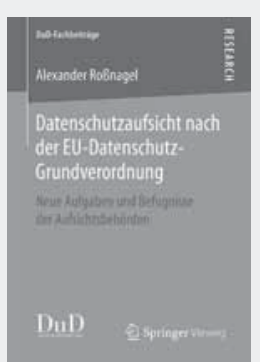

DuD

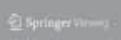
Abb in Farbe Brosch. $€$ (D) $59,99|€(A) 61,67|$ ${ }^{*} \mathrm{sFr} 62,00$ ISBN 978-3-658-17661-7 $€ 46,99$ | ${ }^{*} \mathrm{sFr} 49,50$ ISBN 978-3-658-17662-4 (eBook)

Informationelle Selbstbestimmung im digitalen Wandel

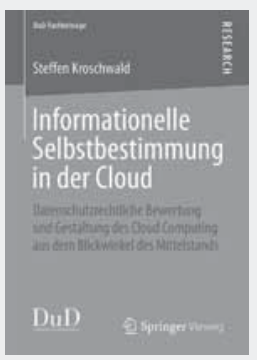

2016, XXIV, $511 \mathrm{~S}$

Brosch. $€$ (D) 79,99|€(A) 82,23| ${ }^{*} \mathrm{sFr} 82,50$ ISBN 978-3-658-11447-3 $€ 62,\left.99\right|^{*} \mathrm{~s} F r$ 66,00 ISBN 978-3-658-11448-0 (eBook)

Informationelle Selbstbestimmung in der Cloud
2017, X, 204 S. Brosch.

$€(D) 49,99|€(A) 51,39|$ ${ }^{*} \mathrm{sFr} 51,50$

ISBN 978-3-658-18505-3 $€ 39,99 \mid{ }^{*} \mathrm{sFr} 41,00$

ISBN 978-3-658-18506-0 (eBook)

Datenschutzaufsicht nach der EU-Datenschutz-Grundverordnung

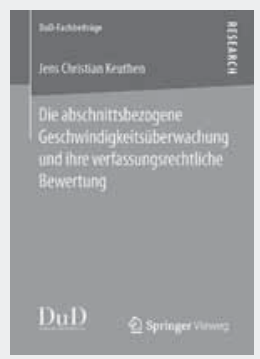

2016, XXIV, 475 S. 1 Abb. Brosch. $€(D) 69,99|€(A) 71,95|$ ${ }^{*} \mathrm{sFr} 72,00$ ISBN 978-3-658-11848-8 $€ 54,99 \mid{ }^{*} \mathrm{~s} F r$ 57,50 ISBN 978-3-658-11849-5 (eBook)

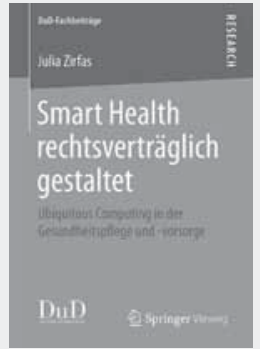

2017, XVI, 243 S. 7 Abb., $3 \mathrm{Abb}$. in Farbe. Brosch. $€$ (D) $54,99|€(A) 56,53|$ ${ }^{*}$ sFr 56,50

ISBN 978-3-658-18349-3 $€ 42,\left.99\right|^{*} \mathrm{sFr} 45,00$ ISBN 978-3-658-18350-9 (eBook)

Smart Health rechtsverträglich gestaltet

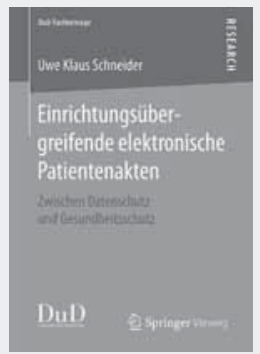

2016, XXVII, 559S.

Brosch.

$€(D) 79,99|€(A) 82,23|$

${ }^{*} \mathrm{SFr} 82,50$

ISBN 978-3-658-11596-8 $€ 62,99 \mid{ }^{*} \mathrm{~s} F \mathrm{Fr} 66,00$ ISBN 978-3-658-11597-5 (eBook)

Die abschnittsbezogene

Geschwindigkeitsüberwachung und ihre verfassungsrechtliche Bewertung

Einrichtungsübergreifende elektronische Patientenakten

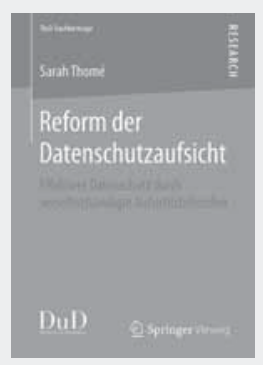

2015, XIII, 153 S. Brosch. $€(D) 59,99|€(A) 61,68|$ ${ }^{*} \mathrm{SFr} 63,50$ ISBN 978-3-658-09752-3 $€ 46,99 \mid{ }^{*} \mathrm{~s} F r 50,50$ ISBN 978-3-658-09753-0

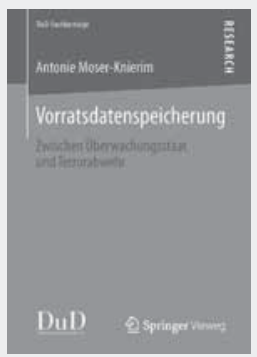
2014, XXVII, 426 S. 1 Abb. Brosch. $€(D) 69,99|€(A) 71,95|$ ${ }^{*} \mathrm{~s} F \mathrm{r} 87,50$ ISBN 978-3-658-04155-7 $€ 54,\left.99\right|^{*} \mathrm{sFr} 70,00$ ISBN 978-3-658-04156-4 (eBook)

Reform der Datenschutzaufsicht
Vorratsdatenspeicherung 
richtigungspflichten führen dabei eine neue Ebene des Spannungsverhältnisses ein. Die Handelnden laufen Gefahr, sich selbst des Datenschutzverstoßes bezichtigen zu müssen. Solche Konstellationen entstehen im Verhältnis der Mitarbeiter zum Arbeitgeber, des Auftragsverarbeiters/Dienstleisters zum Auftraggeber, der Unternehmensleitung gegenüber den Aufsichtsbehörden, usw. Selbst wenn sie für solche Fälle durch weitere Regelungen geschützt sein sollten, stellt dies für den betroffenen Menschen ein inneres Spannungsverhältnis dar. Aus der zivil- und sanktionsrechtlichen Praxis ist bekannt, dass die Hemmschwelle zur proaktiven Mitteilung von haftungs- und sanktionsrelevanten Sachverhalten hoch ist.

Doch gerade vom Umgang mit solchen Spannungsverhältnissen wird abhängen, wie sich die gelebte Kultur der Datenschutz-Grundverordnung entwickeln wird. Die bisherige Praxis der deutschen Aufsichtsbehörden, mit Umsicht und Augenmaß vorzugehen, mag zuweilen kritisiert worden sein. Zugleich gründet auf dieser Vorgehensweise und diesem Selbstverständnis aber auch die Hoffnung, dass das Datenschutzrecht nicht zu einem Sanktionsrecht als Selbstzweck verkommt, sondern der Schutz des Menschen in Bezug auf den Umgang mit seinen Daten im Focus steht und tragender Gedanke ist.

\section{Jens Eckhardt und Konrad Menz}

